\title{
Flotte Senioren
}

\section{Stellen Sie sich auf jung gebliebene Patienten ein!}

\author{
Ute Jürgens
}

Bekanntermaßen sind Klienten über 60 heute nicht nur körperlich 10 Jahre jünger als Gleichaltrige vor einem Jahrhundert. In unseren Köpfen ist allerdings noch das Bild von „damals“ verankert: Alte Menschen seien in vielerlei Hinsicht eingeschränkt.

Wir unterschätzen oft die Fähigkeiten und Kenntnisse der älteren Generation. Sie selbst haben ohnehin meist eine ganz andere Einstellung zum Alter, und die sollten wir kennen. Der Demografieberater und Psychologe Prof. Eberhard G. Fehlau verdeutlicht das steigende Interesse dieser Generation an Gesundheit und lebenslangem Lernen. Statt sich zu ernähren wie ehemals, probieren Ältere gerne andere und neue Wege aus. Sie interessieren sich dafür, wie sie ihre gestiegene Lebenserwartung mit Sport, gesunder Ernährung und Nahrungsergänzungsmitteln unterstützen können [1]. Ein Großteil der Menschen über 60 bleibt länger im Beruf als früher, nicht nur weil offiziell das Rentenalter heraufgesetzt wurde, sondern weil Best Ager noch Lust haben oder aus finanziellen Gründen zur Erwerbsarbeit gezwungen sind. Wer weniger oder gar nicht mehr arbeitet, legt noch lange nicht die Hände in den Schoß, sondern bleibt aktiv, lernt etwas Neues und engagiert sich in Familie und Ehrenamt.

Wir stehen bei der Beratung häufig alten Menschen gegenüber, die sich bereits im Internet bestens informiert haben $(\triangleright$ Abb. 1). So gilt es als erstes, herauszufinden, auf welchem Niveau sie gerade stehen und welcher Spur sie im Netz gefolgt sind. Es ist überflüssig, mit der Beratung ganz vorne anzufangen. Gesucht ist der aktuelle Punkt des Wissens, von dem aus wir starten. Der IT-Branchenverband Bitkom hat ermittelt, dass mittlerweile

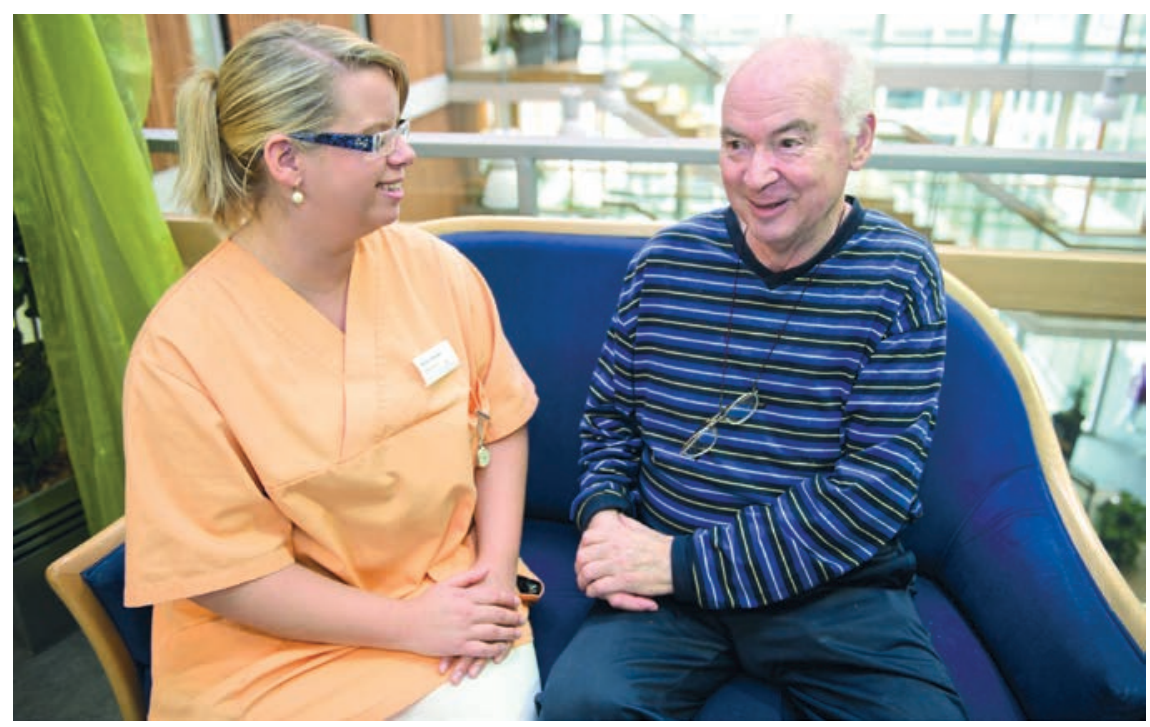

- Abb. 1 Ältere Patienten nicht unterschätzen: Sie sind durchaus gut informiert und erwarten mehr als eine Standardberatung. (C) Thieme Verlagsgruppe/Werner Krüper

knapp 40\% der Menschen über 65 online sind. Sie kaufen hier ein, informieren sich, zahlen ihre Rechnungen online. Das Ganze mit steigender Tendenz. Übrigens sagt auch jeder zweite der Befragten von sich, dass er ohne das Internet nicht mehr auskommen würde [2].

\section{Babyboomer: \\ Klienten mit Kapital}

In Kürze geraten die Babyboomer in die Altersgruppe zwischen 60 und 70, sie sind die zahlenstärkste Generation und in diesem Sinne die Hauptzielgruppe. Eigenartigerweise wird das von vielen Unternehmen ignoriert. Grund dafür ist vermutlich, dass es sich um eine neue, nie dagewesene Entwicklung handelt, die noch außerhalb unserer Vorstellung liegt. Nach wie vor geistert noch das Klischee „Bei den Alten ist ja eh nichts zu holen!“ durch die Köpfe. Nach den Autoren
Muthers und Ronzel besitzt die Gruppe $50+$ jedoch $50 \%$ des verfügbaren Einkommens und 75\% aller Vermögenswerte. Sie kauft 80\% der Luxusautos und 50\% aller Kosmetik [3]. Eine selbst bezahlte Ernährungsberatung, vielleicht mit mehreren Terminen und Themen, ist für viele durchaus möglich. An diesem Punkt möchte ich Sie ermutigen, Ihre Leistung nicht unter Wert anzubieten. Es geht nicht nur um die Zeit, die Sie investieren, sondern auch um die Energie, die Sie einsetzen und Ihr Wissen. Oft setzen Sie sich bei einer privat bezahlten Sitzung mehr ein, als bei Ihrer Standardkrankenkassenleistung. Sie stehen nicht unter Druck, sondern können aus dem Vollen schöpfen.

\section{Nicht unterschätzen, sondern wertschätzen}

Noch immer glaube viele, Senioren seien mit Defiziten behaftet, ihre körperlichen 


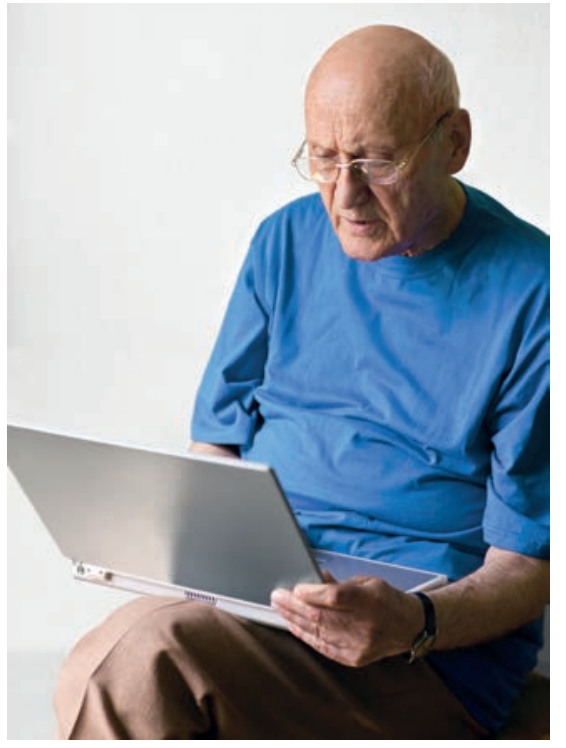

- Abb. 2 Ist Ihre Website aussagekräftig? Sonst geht er woandershin. @ ccvision

Fähigkeiten seien eingeschränkt, ihre Auffassungsgabe sei verlangsamt. Für selbstständige Ernährungsfachkräfte hat das mehrere Konsequenzen: Wenn wir das Gespräch mit einem älteren Menschen beginnen, hinterfragen wir am besten unsere innere Einstellung und beobachten uns selbst: Sprechen wir automatisch lauter, obwohl es nicht nötig ist? Gehen wir davon aus, dass das Gegenüber begriffsstutzig ist oder uninformiert? Wir verlieren Zeit und langweilen den Kunden, wenn wir elementare Dinge erklären, die er längst weiß.

Viele Best Ager werden kurz abgefertigt, Wünsche werden auf diese Weise nicht erkannt und schon gar nicht befriedigt. Wer erkennen lässt, dass er von Langsamkeit oder als so empfundener Umständlichkeit genervt ist, lädt geradezu ein, es das nächste Mal bei einem anderen Berater zu versuchen. Gerade weil niemand zum alten Eisen gezählt werden möchte, entwickelt man sehr feine Antennen für die Ausstrahlung des Beraters. Wer dies nicht beachtet, wird als arrogant, herablassend und desinteressiert erlebt. In meinen Seminaren wird immer wieder von Kunden berichtet, die gewechselt haben. Darauf angesprochen erklären sie: „Bei Ihnen wurde ich immer behandelt wie ein dummer Junge, das lasse ich mir nicht gefallen“ oder „Ich bin doch nicht dement, nur weil ich über 80 bin!“

\section{Sind Sie eingerichtet?}

Ein anderer Punkt erfordert ebenfalls Beachtung: Die Einrichtung des Beratungsraums, Hinweisschilder, Zeitschriften und ähnliche Äußerlichkeiten. Ansprechend ist ein Arbeitsplatz mit verschiedenen Sitzgelegenheiten. Ob Sie dort einen Greyhopper bedienen, der gerade vom Inlineskaten kommt, eine Hochschwangere, Menschen mit Kreislaufbeschwerden oder tatsächlich körperlich schwache alte Menschen: Probieren Sie es aus, manche sitzen am liebsten auf einem Hocker, Ball, Schwingstuhl etc.

\section{Prüfen Sie Ihre Internetpräsenz}

Noch ein dritter besser zu pflegender Bereich ist Ihre Internetseite. Haben Sie Ihre aktuellen Angebote dort verankert? Stellen Sie dort mit oder ohne Passwort Vorträge ein, die Sie in Betrieben oder Sportvereinen gehalten haben? Diejenigen, die noch einmal nachlesen möchten, sind vermutlich nicht sorglose Zwanziger, sondern lebenslustige Sechziger. Alles sollte übersichtlich gegliedert, groß genug geschrieben und schnell zu finden sein.

Ist man nicht mehr gut zu Fuß oder vorübergehend durch Krankheit gehandicapt, ist es eine große Erleichterung, wenn der Ernährungscoach auch Hausbesuche macht. Natürlich sollte auf Ihrer Seite die Möglichkeit zum Bezahlen bestehen, per Lastschrift, Rechnung, Vorauszahlung - auf jeden Fall mehrere Varianten. Eine Firma, die ihre Seite nicht ständig aktualisiert, ist auch bei der älteren Generation nicht mehr gefragt. Sie wird nicht mehr ernst genommen. Dies bemerken wir nicht, niemand sendet eine Mail, in der er kundtut, dass er unzufrieden mit unserem Angebot ist. Nein, jeder sucht sich etwas Besseres ( $\bullet$ Abb. 2).

Sicher können Sie die Nichtakzeptanz des Alterns ein Stück weit nachvollziehen - denken Sie daran, wie viele von uns das Tragen einer Lesebrille hinauszögern! Dabei geht es nicht nur um die Bequemlichkeit, es ist auch nicht so, dass wir uns keine Brille leisten können. Andere starten plötzlich mit Leistungssport oder fordern sich auf andere Weise körperlich heraus. Es ist nicht mehr selbstverständlich, dass alles problemlos funktioniert, das gefällt uns nicht und wir versuchen nun aktiv - oft über das normale Maß hinaus - den Prozess zu verlangsamen.

Ansprechen sollten wir heute alle Kunden auf die gleiche Weise und ein breites Sortiment von Themen anbieten, das alle Altersgruppen berücksichtigt. Ein umfassendes Wissen, was sich auf dem Gesundheitsmarkt befindet, macht sich durchaus bezahlt. Nehmen Sie ältere Menschen ernst, Wertschätzung und Respekt schaffen Vertrauen.

\section{Online}

http://dx.doi.org/10.1055/s-0042-115345

\section{Literatur}

1 Fehlau EG. Erfolgsfaktor 50+. Offenbach: Gabal Verlag; 2011

2 Bitkom e.V. Das Internet wird für viele Senioren unverzichtbar [Presseinformation vom 12.12.2014]. https://www.bitkom.org/ Presse/Presseinformation/Das-Internet-wirdfuer-viele-Senioren-unverzichtbar.html

3 Muthers H, Ronzal W. Marketing 50+. Offenbach: Gabal Verlag; 2012

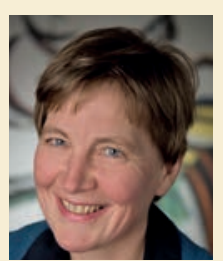

Ute Jürgens KomMed Kommunikationstraining und Einzelcoaching Peter-SonnenscheinStr. 59 28865 Lilienthal

Ute Jürgens arbeitet seit 1981 als PTA und hat Erwachsenenbildung studiert. Die Diplomarbeit untersuchte das Thema Therapiemotivation. Ihre Schwerpunkte sind Einzelcoaching und Persönlichkeitstraining. Sie bietet seit 2004 eigene zertifizierte Seminare bei Bremen an, bundesweit Inhouse-Seminare und wird von Heilberufskammern und Kongressveranstaltern als Referentin gebucht.

info@kommed-coaching.de 